\title{
Sulla curvatura delle faccette di una varietà kähleriana.
}

\author{
Memoria di G. B. Rizza (a Roma)
}

\begin{abstract}
Sunto. - La conoscenea delle curvature (ordinaria e mista) delle faccette caratteristiche di una varietà kähleriana consente la determinazione della curvatura di tntte le faccette delle varietà.
\end{abstract}

1. In diversi lavori (') E. Martinelul ha posto in lace che l'elemento geometrico fondamentale nello studio di una varieta a struttura complessa $V_{2 n}(n>1)$ è il sistema delle $\infty^{2 n-2}$ frccette caratteristiche tangenti nei singoli punti di $V_{2 n}$ (immagini reali delle direzioni complesse) $\left({ }^{2}\right)$. Appare percio naturale, in ogni questione geometrica su $V_{2 n}$, porre in relazione gli enti che interessano con l'insieme delle faccette caratteristiche.

In quest'ordine di idee va considerato il presente lavoro, nel quale mostro che la curvatura delle faccette non caratteristiche di una $\mathrm{V}_{2 n}$ kähleriana dipende unicamente dalle curvature (ordinarie $e$ miste $\left({ }^{3}\right)$ ) delle faccette caratteristiche $d i \mathrm{~V}_{2 n}$ (n. 4).

Il risultato si precisa nel modo seguente. Ad ogni faccetta non caratteristica $E_{2}$ di $V_{2 n}$, come ho indicato in una precedente nota ( $\left.{ }^{*}\right)$, può essere associato un sistema $\infty^{1}$ di faccette caratteristiche (sistema $\Sigma$ ), che permette di definire la deviazione caratteristica di $E_{2}$, ciò̀ il divario della faccetta $E_{2}$ dall' essere caratteristica (n. 3). Ciò, premesso, la curvatura $\mathrm{K}$ di una faccetta $\mathrm{E}_{2}$ di $\mathrm{V}_{2 n}$ si esprime linearmente nelle medie $\mu_{\Sigma}, \mathscr{T}_{\Sigma}$ delle curvature (ordinarie o miste) relative alle faccette caratteristiche del sistema $\Sigma$, associato ad $E_{2}$; precisamente $K=3 \mathfrak{N}_{\Sigma}-2 \mu_{\Sigma}$ (n. 4).

Al n. 5 è segnalata una seconda espressione per la curvatura $K$ di $E_{2}$, nella quale intervengono soltanto le curvature di quattro particolari piani di $\Sigma$.

(1) Ved. i nn. [6], [7], [8], [9], [10] della bibliografia.

(2) L' osservazione sussiste più in generale con riferimento alle varietà quasi-complesse (ved. E. Martinelui, [8], n. 8), alle quali possono di conseguenza venir estesi i risultati del seguito.

(3) La nozione di curvatura mista per una coppia di faccette è stata introdotta da E. Bompiani in [3], p. 130.

(4) G. B. RizzA, [11]. 
Il n. 6 comprende invece l' esame di alcuni casi particolari, tra i quali ha interesse sopra tutto quello di isotropia caratteristica (caso $B$ ) $\left(^{5}\right)$. In questa ipotesi, la curvatura $\mathrm{K}$ di una faccetta $\mathrm{E}_{2}$ dipende soltanto dalla curvatura caratteristica costante $\mathrm{k}$ e dalla deviazione caratteristica $\delta$ di. $\mathrm{E}_{2}$, secondo la formula $K=\frac{k}{4}\left(1+3 \cos ^{2} \delta\right)$. La relazione permette, in particolare, di ritrovare, con maggiori precisazioni, due teoremi di S. BooHNER $\left({ }^{6}\right)$.

2. Generalità. - Sia $V_{2 n}(n>1)$ una varieta kahleriana, $O$ un suo punto $e$ :

$$
d s^{2}=2 g_{\alpha \bar{\beta}} d z^{\alpha} d z^{\bar{\beta}} \quad(\alpha, \beta=1, \ldots, n)
$$

la forma quadratica che definisce la metrica in un intorno $U$ di $O\left({ }^{7}\right)\left({ }^{8}\right)$.

I vettori $u, v, w, \ldots$ di $V_{2 n}$ che si considerano, sono vettori uscenti da $O$.

Sia $J$ la trasformazione lineare nello spazio affine tangente di $O$, che definisce la struttura quasi-complessa, determinata dalla struttura complessa di $V_{2 n}\left({ }^{2}\right)$. Le componenti complesse $\left({ }^{30}\right)$ del vettore $\tilde{u}=J u$, corrispondente al vettore $u$; sono:

$$
\tilde{u}^{\alpha}=i u^{\alpha}, \quad \tilde{u}^{\bar{p}}=-i u^{\bar{\beta}}
$$

quindi $u$ ed $\tilde{u}$ sono ortogonali e di ugual lunghezza.

Una coppia ordinata $u v$ di vettori di $V_{2 n}$ uscenti da $O$ definisce una faccetta di $V_{2 n}$ di origine $O$. Le faccette caralteristiche sono le faccette unite nella trasformazione $J\left({ }^{11}\right)$.

Per un vettore, uscente da $O$, passa una ed una sola faccetta caratte. ristica. Ad es. $u$ determina la faccetta caratteristica $u \tilde{u}$.

(5) Sulle $\nabla_{2 n}$ kulhleriane dotate di isotropia caratteristica ved. S. Bochner, [2], p. 184 K. Yano - S. Bochner, [13], p. 128; E. Martinell, [7], pag. 20 e [10]

(6) S. Bochner, [2].

(7) Per la definizione, le proprietà essenziali, le notazioni ved. K. Yano $\cdot$ S. Bochner, [13], Cap. VIII, nn. 1 e 2 .

(8) Più generalmente si potrebbero considerare varietà quasi-complesse (con o senza torsione), dotate di metrica kähleriana (non necessariamente definita positiva). Ved. le osservazioni al n. 8 del lavorn [8] di E, Martinelli.

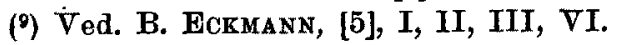

(10) Le componenti complesse del rettore $s$ di componenti reali $\sigma^{j}(j=1, \ldots, 2 n)$ sono $s^{\alpha}=\sigma^{\alpha}+i \nu^{n}+\alpha, s^{\vec{\beta}}=\sigma \beta-i \sigma^{n+\beta}(x, \beta=1, \ldots, n)$.

(ii) In K. Yano-S. Bochner, [13] i termini : 2-dimensional section, holomorphie section equivalgono risp. ai termini qui usati: faccetta, faccetta caratteristica. 
Nel seguito interviene frequentemente il tensore di curvatura $R_{j h k l}$ $(j, h, k, l=1, \ldots, n, \overline{1}, \ldots, \bar{n})$, che gode di note proprieta di simmetria ed emisimmetria.

Conviene notare che, per nna $V_{2 n}$ kähleriana $\Theta$ :

$$
R_{\alpha \bar{\beta} \gamma \bar{\delta}}=R_{\alpha \bar{\delta} \gamma \bar{\beta}}
$$

onde $R_{j h k l}$ risulta simmetrico rispetto agli indici, primo e terzo, secondo e quarto $\left.{ }^{12}\right)$.

È classica la definizione di curvatura di una faccetta. Più in generale, per una coppia di faccette $E$. Bomprani ha introdotta la nozione di curvatura mista $\left({ }^{13}\right)$.

La curvatura della faccetta definita dai vettori unitari e ortogonali $u v \dot{\theta}$ :

$$
K_{u v}=-R_{\alpha \bar{\beta} \gamma}{ }^{\gamma}\left(u^{\alpha} v^{\bar{\beta}} u r v^{\bar{\delta}}-2 u^{\alpha} u^{\bar{\beta}} v^{\gamma} v^{\bar{\delta}}+v^{\alpha} u^{\bar{\beta}} v^{\gamma} u^{\bar{\delta}}\right) .
$$

In particolare, la ourvatura della faccetta caratteristica determinata dal vettore unitario $w$ è:

$$
K_{w \tilde{w}}=4 R_{\alpha \bar{\beta} \gamma \bar{\delta}} w^{\alpha} w^{\bar{\beta}} w r w^{\bar{\delta}}
$$

La curvatura di BOMPIANI per le faccette definite dai vettori $u v, x y$ unitari e ortogonali $\theta$ :

$$
\chi_{u v, \infty \nu}=-R_{\alpha \overline{\bar{\beta}} \bar{\gamma} \bar{\delta}}\left(u^{\alpha} v^{\bar{\beta}} x_{x} y^{\bar{\delta}}-u^{\alpha} v^{\bar{\beta}} y^{r} x^{\bar{\delta}}-v^{\alpha} u^{\bar{\beta}} x y^{\bar{\delta}}+v^{\alpha} u^{\bar{\beta}} y^{r} x^{\bar{\delta}}\right) .
$$

In particolare, la curvatura mista per le faccette caratteristiche determinate dai vettori unitari $r, s$ è:

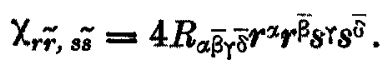

La (6) si ottiene direttamente esplicitando la definizione di curvatura mista $\left({ }^{24}\right)$. Dalla (6), nell' ipotesi che le due faccette coincidano, tenuta presente la (3), scende la (4) $\left({ }^{15}\right)$. In particolare se le faccette sono caratteristiche, dalla (7) segue la (5).

(12) Ved. S. Bochner, [1], p. 788.

(13) E. Bompiani, [B], p. 130.

(ii) Ved. p. es. E. CARTan, [4], p. 195.

(15) Alla (4) si perviene anche direttamente partendo dalla (8.37) di [13] e tenendo conto della (3). In particolare la (5) si ottiene subito dalla (8.40). 
Evidentemente le nozioni introdotte dipendono dalle faccette e non dalle coppie di vettori (unitari e ortogonali) ehe le definiscono. Gli indici bassi nelle notazioni $\bar{K}_{w \tilde{w}}, \chi_{u v, x y}, \chi_{r \tilde{r}, s \tilde{s}}$ servono soltanto per individuare le faccette.

3. Il sistema $\Sigma$. - Sia $E_{2}$ una faccetta non caratteristica di origine $O$, definita dai vettori unitari o ortogonali $u v$ e $\mathbf{\Sigma}$ il sistema $\infty^{1}$ delle faccette caratteristiche individuare dai vettori uscenti da 0 ed appartenenti ad $\mathrm{E}_{2}$.

Le faccette caratteristiche di $\Sigma$ formano con $E_{2}$ angolo costante $\delta$ che dicesi deviazione caratteristica della faccetta $E_{\mathrm{z}} . E^{\prime}$ :

$$
\cos \delta=-u \times \tilde{v}{ }^{\left({ }^{16}\right)} \text {. }
$$

Il sistema $\Sigma$ associato ad $E_{2}$ ha ruolo preminente nel seguito. E utile quindi lo studio delle curvature (ordinaria e mista) delle faccette caratteristiche di $\Sigma$.

Sia $w$ un vettore unitario uscente da $O$ ed appartenente alla faccetta $\boldsymbol{E}_{2} . \boldsymbol{E}^{\prime}$

$$
w=u \cos \tau+v \sin \tau \quad(0 \leq \tau<2 \pi) .
$$

Tenuto conto delle (5) (9) (3), la curvatura della faccetta caratteristica $w \tilde{w}$ di $\Sigma$ è :

$$
\begin{aligned}
K_{w \tilde{w}}= & 4 R_{a \bar{\beta} \bar{\gamma} \bar{\delta}}\left[u^{\alpha} u^{\bar{\beta}} u r u^{\bar{\delta}} \cos ^{4} \tau+2\left(v^{\alpha} u^{\bar{\beta}} u r u^{\bar{\delta}}+u^{\alpha} v^{\bar{\beta}} u r u^{\bar{\delta}}\right) \cos ^{8} \tau \sin \tau+\right. \\
& +\left(u^{\alpha} v^{\bar{\beta}} u v^{\bar{\delta}}+4 u^{\alpha} u^{\bar{\beta}} v v^{\bar{\delta}}+v^{\alpha} u^{\bar{\beta}} v r u^{\bar{\delta}}\right) \cos ^{2} \tau \sin ^{2} \tau+ \\
& \left.+2\left(u^{\alpha} v^{\bar{\beta}} v r v^{\bar{\delta}}+v^{\alpha} u^{\bar{\beta}} v r v^{\bar{\delta}}\right) \cos \tau \sin ^{3} \tau+v^{\alpha} v^{\bar{\beta}} v r v^{\bar{\delta}} \sin ^{4} \tau\right] .
\end{aligned}
$$

La media delle curvature dei piani caratteristici del sistema $\Sigma$ è dunque:

$$
\begin{gathered}
\mu_{\Sigma}=\frac{1}{2 \pi} \int_{0}^{2 \pi} K_{w \tilde{v}} d \tau=\frac{1}{2} R_{\alpha \bar{\beta} \gamma \delta}\left(3 u^{\alpha} u^{\bar{\beta}} u ̛ u^{\bar{\delta}}+3 v^{\alpha} v^{\bar{\beta}} v r v^{\bar{\delta}}+\right. \\
\left.+u^{\alpha} v^{\bar{\beta}} u \curlyvee v^{\bar{\delta}}+4 u^{\alpha} u^{\bar{\beta}} v \curlyvee v^{\bar{\delta}}+v^{\alpha} u^{\bar{\beta}} v \curlyvee u^{\bar{\delta}}\right) .
\end{gathered}
$$

(16) Ved. G. B. Rizza, [11], n. 3. Ivi il sistema $\Sigma$ e la deviazione caratteristica di una faccetta sono considerati per la prima volta. Pì̀ in generale per la definizione di deviazione caratteristica di un elemento $2 q$-dimensionale orientato $E_{2 q}(q=1, \ldots, n)$ ved. G. B. RizzA [12]. 
G. B. Ruzza: Sulla curvatura delle faccette di una varietà kähleriana cioè:

$$
r=u \cos \varphi+v \sin \varphi, \quad s=u \cos \psi+v \sin \psi
$$

$(0 \leq \varphi, \psi<2 \pi)$

Tenuto conto delle (7) (12), la curvatura di BompIanI per le faccette caratteristiche $r \tilde{r}, \tilde{s} \tilde{s}$ di $\Sigma$ è:

$$
\begin{gathered}
\chi_{r \tilde{r}, \tilde{s}}=4 R_{\alpha \overline{\beta_{\gamma}} \bar{\delta}}\left[u^{\alpha} u^{\bar{\beta}} \cos ^{2} \varphi+\left(u^{\alpha} v^{\bar{\beta}}+v^{\alpha} u^{\bar{\beta}}\right) \cos \varphi \sin \varphi+v^{\alpha} v^{\bar{P}} \sin ^{2} \varphi\right] \cdot \\
\cdot\left[u r u^{\bar{\delta}} \cos ^{2} \psi+\left(u r v^{\bar{\delta}}+v^{r} u^{\bar{\delta}}\right) \cos \psi \sin \psi+v v^{\bar{\delta}} \sin ^{2} \psi\right] .
\end{gathered}
$$

Dalle (13) (3) segue che la media delle curvature miste per le faccette caratteristiche di $\Sigma \grave{e}$ :

$$
\mathfrak{T K}_{\Sigma}=\frac{1}{4 \pi^{2}} \iint \chi_{r \tilde{r}, \tilde{s} \tilde{\delta}} d \varphi d \psi=R_{\alpha \bar{\beta} \bar{\delta} \bar{\delta}}\left(u^{\alpha} u^{\bar{\beta}} u r u^{\bar{\delta}}+2 u^{\alpha} u^{\bar{\beta}} v r v^{\bar{\delta}}+v^{\alpha} v^{\bar{\beta}} v r v^{\bar{\delta}}\right)
$$

ove è inteso di integrare da $O$ a $2 \pi$ rispetto a ciascuna variabile.

4. Teorema sulla curvatura - Le considerazioni del a. 3 permettono di dare una semplice espressione della curvatura di una faccetta non caratteristica.

Sia $E_{2}$ una faccetta non caratteristica, definita da una coppia $u v$ di vettori unitari e ortogonali e $\Sigma$ il sistema associato ad $E_{2}$ (n. 3).

Per la curvatura $\mathrm{K}$ della faccetta $\mathrm{E}_{2}$ sussiste la relazione:

$$
K=3 \mathscr{T} \kappa_{\Sigma}-2 \mu_{\Sigma},
$$

dove $\mu_{\Sigma}$ ed $\mathfrak{K}_{\Sigma}$ sona risp. le medie delle curvature ordinarie e miste delle faccette di $\Sigma$.

Alla (15) si perviene direttamente in viıtù delle (4) (11) (14).

E opportuno segnalare che dal risultato stabilito seguono:

a) La curvatura di una faccetta non caratteristica dipende soltanto dalle curvature (ordinarie e miste) delle faccette caratteristiche del sistema $\mathbf{Z}$ associato alla faccetta considerata.

b) La conoscenza delle curvature (ordinarie e miste) delle faccette caratteristiche di origine $O$ è sufficiente a determinare la curvatura di ogni faccetta di origine $O$.. 
5. Osservazioní complementari. - La valutazione delle medie $\mu_{\Sigma}, \mathfrak{T K}_{\Sigma}$, che intervengono nella relazione (14), puo ottenersi nel modo seguente.

Sulla faccetta $E_{2}$ si considerino due rette ortogonali per $O$ e le relative bisettrici. Siano $u v, x y$ vettori unitari nscenti da $O$ od aventi risp. le dire. zioni delle due coppie di rette considerate.

Cio premesso, vale la relazione:

$$
\mu_{\Sigma}=\frac{1}{4}\left(k_{\boldsymbol{u} \tilde{u}}+k_{v \tilde{v}}+k_{x \tilde{x}}+k_{y \tilde{y}}\right)
$$

che proviene dalla (10), assegnando successivamente all'angolo $\tau$, che interessa mod. $\pi$, i valori $0, \frac{\pi}{4}, \frac{\pi}{2}, \frac{3 \pi}{4}$.

Analogamente, dalle (5) (7) scende la relazione:

$$
\operatorname{TK}_{\Sigma}=\frac{1}{4}\left(k_{u \tilde{u}}+2 \chi_{u \tilde{u}, \tilde{v}}+k_{v \tilde{v}}\right) \text {. }
$$

Infine, la curvatura $\mathrm{K}$ di una faccetta non caratteristica $\mathrm{E}_{2}$ di origine 0 pud esprimersi mediante le curvature (ordinarie e miste) di quattro faccette caratteristiche di $\Sigma$, le quali incontrino $\mathrm{E}_{2}$ secondo due rette ortogonali per 0 e le loro bisettrici. Precisamente:

$$
K=-\frac{1}{8}\left(k_{u \tilde{u}}+k_{v \tilde{v}}+k_{x \tilde{x}}+k_{y \tilde{y}}-6 \chi_{u \tilde{u}, v \tilde{v}}-6 \chi_{x \tilde{x}, y \tilde{y})} .\right.
$$

Alla (18) si perviene partendo dalle (15) (16) (17) e notando che nella (17) la coppia di vettori unitari ortogonali $u v$ può sostituirsi con la coppia $x y$.

6. Casi particolari. - Si considerano alouni casi particolari, che si presentano imponendo alle curvature delle faccette di origine $O$ ipotesi via via più forti.

A) L-isotropia: la curvatura delle faccette caratteristiche del sistema $\Sigma$ associato alla faccetta $E_{2}$ è costante. Quindi:

$$
K_{w \tilde{w}}=k_{\mathrm{\Sigma}}=\text { costante }
$$

al variare di $\tau$ nella (9), vale a dire al variare del vettore unitario $w$ nel fascio di centro $O$ su $E_{2}$.

Le (16) (17) divengono:

$$
\mu_{\Sigma}=k_{\Sigma}, \quad \mathfrak{M K}_{\Sigma}=\frac{1}{2}\left(k_{\Sigma}+\chi_{m \tilde{u}, \tilde{v}}\right)
$$


Infine, la curvatura $\mathrm{K}$ della $\mathrm{E}_{2}$ è espressa dalla relazione:

$$
K=\frac{1}{2}\left(3 \chi_{u \tilde{u}, \tilde{v}}-k_{\mathrm{g}}\right)
$$

che segue dalle (15) (20) o direttamente dalla (18) $\left({ }^{17}\right)$.

Nell'ipotesi di $\Sigma$-isotropia non $\otimes$ difficile esaminare la distribuzione delle curvature di BOMPIANI relative alle faccette di $\Sigma$.

Posto:

$$
\begin{gathered}
\mathfrak{Q}=4 R_{\alpha \bar{\beta} \gamma \bar{\delta}}\left(u^{\alpha} v^{\bar{\beta}}+v^{\alpha} u^{\bar{\beta}}\right) u r u^{\bar{\delta}}, \quad \mathscr{B}=4 R_{\alpha \bar{\beta} \gamma \bar{\delta}}\left(u^{\alpha} v^{\bar{\beta}}+v^{\alpha} u^{\bar{\beta}}\right) v r v^{\bar{\delta}}, \\
\mathcal{C}=4 R_{\alpha \bar{\beta} \gamma \bar{\delta}}\left(u^{\alpha} v^{\bar{\beta}} u r v^{\bar{\delta}}+4 u^{\alpha} u^{\bar{\beta}} v^{r} v^{\bar{\delta}}+v^{\alpha} u^{\bar{\beta}} v r u^{\bar{\delta}}\right),
\end{gathered}
$$

le (10) (13) del n. 3 divengono

$$
k_{v \tilde{w}}=k_{u \tilde{u}} \cos ^{4} \tau+k_{v \tilde{v}} \sin ^{4} \tau+\mathcal{C} \sin ^{2} \tau \cos ^{2} \tau+\sin 2 \tau\left(\mathfrak{Q} \cos ^{2} \tau+\mathscr{B} \sin ^{2} \tau\right)
$$$$
\chi_{r \tilde{r}, \tilde{s}}=k_{u \tilde{u}} \cos ^{2} \varphi \cos ^{2} \psi+k_{v \tilde{v}} \sin ^{2} \varphi \sin ^{2} \psi+\mathcal{C} \cos \varphi \cos \psi \sin \varphi \sin \psi+
$$$$
+\sin (\varphi+\psi)[\mathcal{Q} \cos \varphi \cos \psi+\mathscr{B} \sin \varphi \sin \psi]+\sin ^{2}(\varphi-\psi) X_{\text {uน } \tilde{u}, \tilde{v}} \text {. }
$$

Assegnati successivamente a $\tau$ nella $(23)$ i valori $\frac{\pi}{4},-\frac{\pi}{4}$, per somma e differenza delle uguaglianze ottenute, nell'ipotesi (19), risulta:

$$
\mathfrak{C}=2 k_{\Sigma}, \quad \mathfrak{a}+\mathfrak{B}=0 .
$$

In virtù delle (19) (25), dalla (23) segue $\mathfrak{Q} \sin 2 \tau \cos 2 \tau=0$ con $\tau$ ąrbitrario; quindi :

$$
\mathfrak{A}=\mathfrak{B}=0 \text {. }
$$

In conclusione, per la curvatura mista delle faccette di $\mathbf{\Sigma}$ sussiste la relazione:

$$
\chi_{r \tilde{r}, s \tilde{s}}=k_{\Sigma} \cos ^{2}(\varphi-\psi)+\chi_{u \tilde{u}, v \tilde{v}} \sin ^{2}(\varphi-\psi)
$$

che deriva direttamente dalla (24), tenuto conto delle (19) (25) (26).

(27) Come appare dalla $(20)_{2} \chi_{r \tilde{r}, s \tilde{s}}$ e costante per ogni coppia $r$ di vettori unitari ortogonali di $E_{2}$ uscenti da 0 . 
I valori estremi di $\chi_{r \tilde{r}}, \tilde{s}$, al variare delle facuette in $\Sigma$, sono $k_{\Sigma}$ e $\chi_{\mu \tilde{u}, \tilde{v}}$ $\theta$ si ottengono in corrispondenza a vettori $r, s$ risp. coincidenti e ortogonali.

B) Isotropia caratteristica: la curvatura delle faccette caratteristiche di origine $O$ è costante. Per ogni vettore unitario $w$ uscente da $O$ è quindi:

$$
K_{w \tilde{w}}=k=\text { costante. }
$$

Dalle (5) (28) deriva la relazione:

$$
R_{\alpha \bar{\beta} \bar{\gamma} \bar{\delta}}=\frac{k}{2}\left(g_{\alpha \bar{\beta}} g_{\gamma \bar{\delta}}+g_{\alpha \bar{\delta}} g_{\curlyvee \bar{\beta}}\right) \quad\left({ }^{18}\right)
$$

Indipendentemente dall'ipotesi di isotropia caratteristica per due vettori arbitrari $a, b$ può scriversi :

$$
a * b=g_{\mu \nu \bar{\nu}} a b^{\bar{\nu}}=\frac{1}{2}[(a \times b)+i(a \times \tilde{b})] .
$$

Sia ora $E_{2}$ una faccetta di origine $O$, definita dai vettori unitari ortogonali $u v, \Sigma$ il sistema associato ad $E_{2}, \delta$ la deviazione caratteristica di $E_{2}(n .3)$.

Dalle (30) (8) segue:

$$
u * v=g_{\mu \nu} u^{\mu} v^{\nu}=-\frac{i}{2} \cos \delta .
$$

Tenuto poi conto delle (29) (31), la (7) diviene:

$$
\chi_{u \tilde{u}, \tilde{v}}=\frac{k}{2}\left(1+\cos ^{2} \delta\right)
$$

onde la (20) si riduce a:

$$
\mu_{\Sigma}=k, \quad 9 \kappa_{\Sigma}=\frac{k}{4}\left(3+\cos ^{2} \delta\right) .
$$

Infine, nell' ipotesi di isotropia caratteristica, per la curvatura $\mathrm{K}$ della faccetta $\mathrm{E}_{2}$ sussiste la relazione:

$$
K=\frac{k}{4}\left(1+3 \cos ^{2} \delta\right),
$$

che scende direttamente dalle (21) (28) (32).

(18) Ved. K. Yano = S. Bochner, [13], p. 128. 
I valori estremi $K$ sono $k$ e $\frac{k}{4}$ e si ottengono risp. per $\delta \equiv 0, \frac{\pi}{2}$ (mod. $\pi$ ), vale a dire in corrispondenza di faccette caratteristiche $\theta$ di faccette a pro. dotto hermitiano reale $\left({ }^{19}\right)$. Questo risultato, immediata conseguenza della (34), precisa un teorema di $\mathrm{S}$. BochNer $\left({ }^{20}\right)$.

Per le curvature miste delle faccette di $\Sigma$ sussiste la relazione:

$$
\chi_{r \tilde{r}, \tilde{B}}=\frac{k}{2}\left(2-\sin ^{2} \lambda \sin ^{2} \delta\right) \quad(\lambda=\varphi-\psi)
$$

che segue dalla (27), in virtù delle (28) (32).

I valori estremi di $\chi_{r \tilde{r}}, s \tilde{s}$ sono $k$ e $\frac{k}{2}\left(1+\cos ^{2} \delta\right)$ e si ottengono risp. per $\lambda \equiv 0, \frac{\pi}{2}(\bmod . \pi)$.

Eे bene notare che la (35) esprime, più generalmente, la curvatura di BOMPIANI per una coppia qualunque di faccette caratteristiche di origine 0. Basta intendere che $\mathrm{i}$ vettori unitari $r, s$ uscenti da $O$ definiscano le due faccette in considerazione, $\lambda$ denoti l'angolo $\overline{r s}$ e $\delta$ la deviazione caratteristica della faccetta $r s$.

I valori estremi della curvatura mista per le coppie di faccette caratte. ristiche sono evidentementemente $k$ e $\frac{k}{2}$ e si presentano risp. quando le due faccette coincidono e quando segano una faccetta a prodotto hermitiano reale secondo due vettori per $O$ ortogonali.

Infine, siano $E_{2}, E_{2}{ }^{*}$ due faccette arbitrarie di origine 0 , definite dalle coppie $u v, x y$ di vettori unitari ortogonali uscenti da $O$ e $\delta, \delta^{*}$ le deviazioni caratteristiche di $E_{2}, E_{2}{ }^{*}$ risp.

La curvatura mista relativa alle faccette $\mathrm{E}_{2}, \mathrm{E}_{2}^{*} \grave{e}$ :

$$
\chi_{E_{2} E_{2}^{*}}=\frac{k}{4}\left(\cos E_{2} E_{2}^{*}+2 \cos \delta \cos \delta^{*}+\cos E_{2} \tilde{E}_{2}^{*}\right)
$$

ove $\widetilde{\mathrm{E}}_{2}^{*}=\mathrm{JE}_{2}^{*}$.

La (36) deriva dalla (6) in virtù della (29). Per la dimostrazione vanno tenute presenti le (30) (31) e, per l'angolo di due faccette, le (12) (13) del lavoro [11].

(19) Ved. G. B. Rrzza [11], n. 8.

(20) S. Bochi R, p. 186; ved. anche K. Y YNo - S. Bochner, [13], p. 128. 
C) Isotropia: la curvatura delle faccette di origine $O$ e costante.

Non varia quindi il primo membro della (34) al variare di $\delta$ da 0 a $\frac{\pi}{2}$. Ciò è possibile se e solo se $K=0$, vale a dire se e solo se la curvatura ̀̀ nulla per ogni faccetta di origine 0.

E cosi ritrovato un teorema di S. Bochner ${ }^{21}$ ).

(21) Ved. K. Yano - S. Bochner, [13], p. 127.

\section{BIBLIOGRAFIA}

[1] S. Bochner, Vector fields and Ricci curvature, Bull. Am Math. Soc. ", 52, (1946).

[2] S. Bochner, Curvature in Hermitian manifolds, . Bull. Am. Math. Sac. , 53, (1947).

[3] E. Bompiani, Spazi viemanniani luoghi di varietd totalmente geodetiche, "Rend Palermo s, 48, (1924).

[4] E. Cartan, Leģons sur la géométrie des espaces de Riemann, Gauthier-Villars, Paris, (195̃1).

[5] B. Eckmann, Cours sur les varietes complexes, *Centro Int. Mat. Est., C I. M. E. * (1956), Edizioni Cremonese, Roma.

[6] E. Martinet.LI, Qualche proprietò geometrica elle varietà a struth́ura complessa, * Atti Acc. Ligure, 9, (1952).

[7] D. MARTINelu, Punti di vista geometrici nello studio delle varietà a struttura complessa, - Centro Int. Mat. Est., C. I. M. E. *, (1956), Edizioni Cremonese, Roma.

[8] E. Martinelat Sulle varietà a struttura complessa. Ann. di Mat. ", 43, (1957).

[9] E. MaRTinelLI, Sulla curvatura delle superficie caratteristiche in una varietà kähleriana, : Rend. Lincei, 21, (1956).

[10] E. Martinelu, Sulle varietà dotate di isotropia caratteristica, *Rend. Lincei *, 21, (1050).

[11] G. B. RIzzA, Deviazione caratteristica delle faccette piane di una varietò a struttura complessa, Rend. Lincei *, 24, (1958).

[12] G. B. RizzA, Holomorphic deviation for the 2q-dimensional sections of complex analy. tic manifolds, *Archiv der Math. *, 10, (1959).

[13] K. Yaxo - S. Bochner, Curvature and Betti numbers, .Ann. of Math. Studies ", 32, Princeton Univ. Press, (1958). 